\title{
A atuação da Enfermagem em cirurgias oftalmológicas: relato de experiência
}

\author{
Nursing care in ophthalmologic surgeries: experience report \\ Desempeño de Enfermería en cirugías oftálmicas: relato de experiencia
}

Recebido: 20/08/2021 | Revisado: 26/08/2021 | Aceito: 29/08/2021 | Publicado: 31/08/2021

\author{
Lisiane Paula Sordi Matzenbacher \\ ORCID: https://orcid.org/0000-0002-1407-7717 \\ Hospital de Clínicas de Porto Alegre, Brasil \\ E-mail: 1sordi@hcpa.edu.br \\ Débora Machado Nascimento do Espírito Santo \\ ORCID: https://orcid.org/0000-0003-0533-0335 \\ Hospital de Clínicas de Porto Alegre, Brasil \\ E-mail: dsanto@hcpa.edu.br \\ Rosaura Soares Paczek \\ ORCID: https://orcid.org/0000-0002-4397-1814 \\ Hospital de Clínicas de Porto Alegre, Brasil \\ E-mail: rspaczek@gmail.com \\ Ana Karina Silva da Rocha Tanaka \\ ORCID: https://orcid.org/0000-0003-2488-3656 \\ Universidade Federal do Rio Grande do Sul, Brasil \\ E-mail: anakarinatanaka@gmail.com \\ Carina Galvan \\ ORCID: https://orcid.org/0000-0003-2111-5432 \\ Hospital de Clínicas de Porto Alegre, Brasil \\ E-mail: cgalvan@hcpa.edu.br
}

\begin{abstract}
Resumo:
Objetivo: descrever a experiência vivenciada pelas enfermeiras, em um centro cirúrgico ambulatorial, durante as fases de atendimento a pacientes submetidos a cirurgias oftalmológicas. Método: estudo descritivo, do tipo relato de experiência, narrado por enfermeiras assistenciais com vivência profissional no centro cirúrgico ambulatorial que presta cuidados a pacientes no perioperatório de cirurgias oftalmológicas. O campo do estudo é um hospital público universitário, localizado na região Sul do Brasil. Resultados: no dia do agendamento da cirurgia, o paciente é admitido no centro cirúrgico, acompanhado de um responsável maior de 18 anos. O técnico de Enfermagem realiza o primeiro atendimento, colocando a pulseira de identificação com nome completo e número de prontuário, verificando sinais vitais, peso e altura, coletando informações, como alergias e horário do jejum, uso de algum aparelho, órtese ou prótese móvel. A equipe cirúrgica realiza a marcação da lateralidade; a enfermeira realiza a anamnese e o anestesista faz a avaliação do paciente. A Enfermagem acompanha o paciente durante o transoperatório. Ao término do procedimento, o paciente é encaminhado para sala de recuperação pós-anestésica onde permanece até a sua total recuperação. Quando em condições de alta hospitalar, um plano de cuidados domiciliares é implementado para que o paciente possa realizar os cuidados. Conclusão: o enfermeiro é um facilitador do processo cirúrgico e deve implementar estratégias embasadas em conhecimento técnico-científico, cumprindo os padrões de qualidade e segurança, trazendo maior qualidade da assistência prestada aos cuidados de Enfermagem, estimulando o desenvolvimento do raciocínio crítico relacionado à temática.
\end{abstract}

Palavras-chave: Procedimentos cirúrgicos oftalmológicos; Cuidados de enfermagem; Cuidados pós-operatórios.

\begin{abstract}
Objective: describe the experience lived by nurses in an ambulatory surgical center during the phases of care of patients undergoing ophthalmologic surgeries. Method: this is a descriptive study, of the experience report type, narrated by nurses with professional experience in an ambulatory surgical center that provides care to patients in the perioperative period of ophthalmologic surgeries. The field of study is a public university hospital located in the South region of Brazil. Results: on the day the surgery is scheduled, the patient is admitted to the surgical center accompanied by a guardian over 18 years of age. The Nursing technician performs the first care, putting on the identification bracelet with full name and medical record number, checking vital signs, weight and height, collecting information such as allergies and fasting time, use of any device, orthosis or mobile prosthesis. The surgical team performs the laterality marking; the nurse performs the anamnesis and the anesthesiologist performs the patient's evaluation. The nursing team accompanies the patient during the trans-operative period. At the end of the procedure, the patient is taken to the recovery room, where he/she remains until his/her full recovery. When able to be discharged from the hospital, a home care plan is implemented so that the patient can perform the care. Conclusion: the nurse is a facilitator of the surgical process and must implement strategies based on technical-scientific knowledge, complying with quality and safety
\end{abstract}


standards, bringing greater quality of the assistance provided to Nursing care, stimulating the development of critical thinking related to the theme.

Keywords: Ophthalmologic surgical procedures; Nursing care; Postoperative care.

\section{Resumen}

Objetivo: describir la experiencia vivida por enfermeras en un centro quirúrgico ambulatorio durante las fases de atención a pacientes sometidos a cirugía oftálmica. Método: estudio descriptivo, tipo relato de experiencia, narrado por enfermeras clínicas con experiencia profesional en un centro quirúrgico ambulatorio que brinda atención a pacientes en el período perioperatorio de cirugías oftálmicas. El campo de estudio es un hospital universitario público ubicado en el sur de Brasil. Resultados: el día en que está programada la cirugía, el paciente ingresa al quirófano acompañado de un responsable mayor de 18 años. El técnico de Enfermería realiza la primera visita, colocándose el brazalete de identificación con nombre completo y número de historia clínica, comprobando signos vitales, peso y talla, recogiendo información como alergias y tiempo de ayuno, uso de cualquier dispositivo, órtesis o prótesis móvil. El equipo quirúrgico realiza el marcaje de lateralidad; la enfermera realiza la anamnesis y el anestesiólogo evalúa al paciente. La Enfermería acompaña al paciente durante el transoperatorio. Al finalizar el procedimiento, el paciente es trasladado a la sala de recuperación donde permanece hasta su completa recuperación. Cuando se encuentra en condiciones de alta hospitalaria, se implementa un plan de atención domiciliaria para que el paciente pueda realizar la atención. Conclusión: el enfermero es un facilitador del proceso quirúrgico y debe implementar estrategias basadas en el conocimiento técnicocientífico, cumpliendo con los estándares de calidad y seguridad, trayendo mayor calidad de la atención al cuidado de Enfermería, fomentando el desarrollo de razonamientos críticos relacionados con la temática.

Palabras clave: Procedimientos quirúrgicos oftálmicos; Cuidado de enfermera; Cuidado postoperatorio.

\section{Introdução}

As doenças oculares são problemas oftalmológicos provocados por inúmeros motivos, desde causas genéticas até hábitos e estilo de vida, podendo causar dificuldade na visão ou doenças mais graves e cegueira. As principais doenças são: catarata, glaucoma, conjuntivite, retinopatia diabética, degeneração macular relacionada à idade e erros de refração. No Brasil, mais da metade dos gastos em Oftalmologia pelo SUS, é para remunerar apenas as cirurgias de catarata e há filas desde a marcação de consultas nas unidades de saúde. A fila é a porta de entrada do SUS e pode acarretar implicações na visão dos usuários, estando presente na vida de milhões de brasileiros que buscam um atendimento digno e resolutivo (Oliveira \& Meneguin, 2019; Santana et al., 2020).

As cirurgias mais comuns são a facectomia, que consiste na remoção da catarata, exérese de pterígio e cirurgia de glaucoma. Tendo incidência maior em adultos, com idade igual ou superior a 80 anos, e a catarata é responsável por $50 \%$ dos casos de perda total de visão (Costa et al., 2021).

As complicações no pós-operatório manifestam-se em um período tardio, o que demonstra a importância do devido acompanhamento e da realização de múltiplas avaliações durante o tratamento após a cirurgia. Há uma maior frequência em cirurgias de catarata, como o descolamento de retina e de luxação da lente intraocular (Meirelles et al., 2020).

A proteção da visão, por meio da prevenção de problemas oculares, deve ser realizada por meio de consultas periódicas com profissional oftalmologista. Muitas vezes, a capacidade de enxergar o mundo claramente não é valorizada, na maioria das vezes, por medo do desconhecido ou de dificuldade de acesso às informações e assistência à saúde. Alguns estudos indicaram que, quase $80 \%$ das doenças nos olhos poderiam ser evitadas, caso fossem diagnosticadas precocemente. O olho é o órgão do sentido mais importante e altamente especializado e sensível, no qual diversos distúrbios visuais podem levar a um comprometimento da visão, chegando à cegueira (Ribeiro et al., 2017).

Os benefícios que as cirurgias oftalmológicas trazem são inúmeros, pois a perda visual, por diversos tipos de problemas oculares, pode ser resolvida em serviços públicos de saúde que possuam equipe qualificada e especializada para este fim. A manutenção da saúde oftalmológica e a adesão do paciente ao tratamento instituído, estão vinculados com a satisfação e a segurança que ele possui na equipe médica e de Enfermagem (Santana et al., 2020).

Após a indicação do procedimento cirúrgico oftalmológico, o enfermeiro tem o papel fundamental de prestar uma assistência de Enfermagem sistematizada, prezando o cuidado e as orientações que poderão, além de constituir vínculos de 
confiança e promover segurança, prevenir complicações significativas que comprometem uma recuperação pós-anestésica adequada. Portanto, a finalidade de sistematizar as condutas e ações na assistência de Enfermagem são de grande efetividade com a aplicação de Protocolos Operacionais Padrão (POPs) como instrumento para capacitar e alinhar as condutas para toda equipe de Enfermagem, desenvolvendo uma assistência humanizada e segura (Carvalho et al., 2016; Muniz \& Paczek, 2021).

Este estudo tem por objetivo descrever a experiência vivenciada pelas enfermeiras em um centro cirúrgico ambulatorial durante as fases de atendimento a pacientes submetidos a cirurgias oftalmológicas.

\section{Metodologia}

Trata-se de um estudo descritivo, do tipo relato de experiência, realizado em julho de 2021, descrito por enfermeiras assistenciais com a vivência profissional em um centro cirúrgico ambulatorial que presta cuidados a pacientes no perioperatório de cirurgias oftalmológicas. O campo do estudo é em um hospital público universitário localizado na Região Sul do Brasil.

O centro cirúrgico ambulatorial possui oito salas cirúrgicas, sendo duas delas destinadas especificamente a cirurgias oftalmológicas. A sala de recuperação pós-anestésica possui 15 leitos de recuperação adulta e cinco leitos pediátricos.

Este relato é o detalhamento da atuação dessas enfermeiras, especificando o conhecimento a respeito dos cuidados cirúrgicos perioperatórios no cenário oftalmológico, de modo a elucidar e a externar a prática aplicada, auxiliando no entendimento e na compreensão da temática escolhida, compartilhando ideias e realizando a troca de conteúdo para o aperfeiçoamento dos cuidados assistenciais sistematizados na prática da Enfermagem (Daltro \& Farias, 2019).

\section{Resultados}

O centro cirúrgico ambulatorial realiza diversos procedimentos oftalmológicos, como cirurgias oncológicas, oculoplásticas, cataratas, vítreo e retina, transplantes de tecidos, glaucoma, estrabismo e injeção intravítrea, sendo, em sua grande maioria, de caráter ambulatorial. No dia do agendamento da cirurgia, o paciente é admitido no centro cirúrgico, na companhia de um familiar e/ou acompanhante responsável maior de 18 anos. Após ser liberado pelo serviço de secretaria, que confere todos os documentos necessários para o procedimento, dá-se início ao preparo para a cirurgia com a equipe de Enfermagem. O técnico de Enfermagem realiza o primeiro atendimento, colocando a pulseira de identificação com nome completo e número de prontuário, verifica sinais vitais, peso e altura e coleta informações, como alergias e horário do jejum, uso de algum aparelho, órtese ou prótese móvel. Também devem ser retirados os adornos e toda vestimenta, procedendo ao preparo com roupa específica para a cirurgia.

Paciente e acompanhante aguardam juntos na sala de preparo pré-operatória até o momento da ida para a sala cirúrgica, pois é necessário pingar colírios para a dilatação da pupila. Nessa etapa, são realizadas a consulta de Enfermagem, com a enfermeira e, a consulta, com a equipe assistencial e com a anestesiologista. $\mathrm{Na}$ anamnese, itens importantes, como o uso de medicações contínuas, cirurgias e tratamentos prévios, são coletados. A checagem da existência de alguma alergia e a demarcação do sítio cirúrgico são obrigatórias para a continuidade do procedimento. É de responsabilidade da equipe médica cirúrgica, junto com o paciente e seu acompanhante, a realização da marcação da lateralidade do sítio operatório, para que não ocorra nenhum tipo de evento adverso. Essa marcação deve ser no sítio operatório ou o mais próximo possível; é realizada com caneta permanente que não seja removida durante o preparo da pele e é representada por meio de um alvo.

Ao ser encaminhado para sala cirúrgica, o circulante de sala é quem acompanhará o paciente no preparo e o encaminhará de cadeira de rodas, até a sala de cirurgia. Nesse momento, o acompanhante é liberado e orientado a esperar na recepção do centro cirúrgico onde será procurado, ao final do procedimento, pela equipe médica cirúrgica para the passar as informações de como transcorreu o procedimento e as orientações referentes à alta hospitalar e aos retornos. No momento da alta do paciente, essas informações serão orientadas junto ao paciente e seu acompanhante pela enfermeira da sala de recuperação pós anestésica. 
A sala cirúrgica está equipada com mesa cirúrgica, microscópio, televisão, eletro cautério mono e bipolar, carro de anestesia, monitor multiparâmetro, vitreófago utilizado nas cirurgias de vitrectomia e facoemulsificador utilizado para fragmentar a catarata nos procedimentos de facoemulsificação. São realizadas a conferência e a testagem de todo material e dos equipamentos pela equipe de Enfermagem.

O paciente é posicionado em decúbito dorsal, com os braços levemente abduzidos e apoiados em braçadeiras próprias da mesa cirúrgica. A mesa deve ser posicionada de tal forma que fique na posição ideal para a realização da técnica anestésica, deixando a cabeceira livre para a disposição do microscópio, permitindo a abordagem do cirurgião. O paciente que é submetido a procedimentos oftalmológicos apresenta sensibilidade aumentada ao barulho e aos ruídos do ambiente, decorrentes da técnica anestésica, que, normalmente, é anestesia local associada a uma sedação leve. Portanto, o ambiente cirúrgico deve ser silencioso e tranquilo para que diminua a ansiedade e permaneça a capacidade de cooperação do paciente. Nesse momento, é realizada a pausa cirúrgica, uma pausa antes da incisão para a aplicação do checklist de cirurgia segura, que é realizado pelo técnico de Enfermagem circulante de sala.

Essa é uma oportunidade para certificar-se de que o paciente está corretamente posicionado; a lateralidade é confirmada, verificando se todos os materiais e equipamentos estão funcionando e disponíveis para uso. Esta lista de verificação promove a cultura de segurança ao paciente cirúrgico oftalmológico de forma sólida no processo do cuidar e todos os membros das equipes participam: Enfermagem, cirurgião e anestesiologista. O serviço de centro cirúrgico ambulatorial conta com um checklist elaborado especificamente para cirurgias oftalmológicas e injeção intravítrea. O diferencial é certificar-se de que estejam disponíveis itens como colírio, lente intraocular, córnea doadora (nos casos de transplantes) e o medicamento a ser utilizado nos casos das injeções intravítreas.

Se o procedimento for realizado apenas com anestesia local, o paciente é encaminhado, pelo técnico de Enfermagem, à sala de preparo, onde é solicitada a presença do acompanhante para auxiliá-lo a vestir-se e, após, são realizadas as orientações de alta pelo residente da equipe da Oftalmologia que entregará todos os documentos, como sumário de alta, retorno para consulta e receita médica.

A anestesia, nas cirurgias oftalmológicas, é escolhida pelo anestesiologista e o cirurgião de acordo com o procedimento realizado e deve ser efetuada logo após a aplicação do checklist. Quando se opta pela realização de bloqueios peribulbares, o anestesista posiciona o baroftalmo (uma bolsa de mercúrio que pesa em torno de 550 gramas) sobre o olho bloqueado, durante dez a quinze minutos, para diminuir a pressão intraocular e, logo após a anestesia, inicia-se a cirurgia.

Ao término do procedimento realizado com anestesia geral ou sedação associada com bloqueio peribulbar, a equipe de Enfermagem realiza a transferência do paciente da mesa cirúrgica para cama e o encaminha para a sala de recuperação pósanestésica, acompanhado do anestesiologista e técnico de Enfermagem.

Para uma assistência segura na sala de recuperação pós-anestésica algumas condições são necessárias para a alta do pacientedições para a alta do paciente ambulatorial sejam: orientação de tempo e espaço; estabilidade de sinais vitais, pelo menos, em 60 minutos; ausência de náuseas e vômitos; dor controlada; dificuldade respiratória; capacidade de ingerir líquidos e de locomoção igual ao pré-operatório; sangramento operatório mínimo; ausência ou alívio de dor; ausência de sinais de retenção urinária e estar acompanhado de um responsável maior de 18 anos.

A orientação de alta para o paciente no pós-operatório em cirurgia oftalmológica, deve ser realizada pela enfermeira, na presença de um acompanhante do paciente. O paciente deve ser orientado a não realizar esforços, não dormir sobre o olho operado, não abaixar a cabeça ou olhar para baixo, não poderá dirigir naquele dia, evitar se expor ao calor do fogão e manter curativo ocular oclusivo, até o próximo dia em que deverá comparecer no ambulatório para ser avaliado junto à equipe médica e reforçado o retorno ao hospital para atendimento de eventuais intercorrências. A conduta de educação é realizada verbalmente pela enfermeira e entregue formulário por escrito. 


\section{Discussão}

O centro cirúrgico é um setor que demanda conduta imediata, seja para procedimentos terapêuticos, análise diagnóstica, tratamentos e correções cirúrgicas. A montagem da sala cirúrgica configura um importante processo do período perioperatório, necessitando, para isso, de equipe capacitada e com habilidades técnicas. O enfermeiro exerce papel de liderança e deve supervisionar e implantar ações que visam à efetiva segurança durante o ato cirúrgico. A Enfermagem presta assistência em toda fase perioperatória, organiza e planeja a prevenção de complicações físicas e emocionais, promovendo uma recuperação completa e segura do paciente (Souza et al., 2019; Ribeiro \& Cunha et al., 2018).

A cirurgia objetiva aumentar os padrões de qualidade e segurança do cuidado cirúrgico, prevenindo infecções do sítio cirúrgico e mantendo anestesia e equipes seguras. Assim, a Organização Mundial de Saúde (OMS) desenvolveu um checklist da campanha de cirurgia segura, indispensável para garantir maior segurança e menor probabilidade de possíveis erros, gerando uma relação de ganho para todos que participam, pois todos os perigos evitados no processo cirúrgico geram uma economia substancial às instituições de saúde, à sociedade e benefícios intangíveis ao paciente. A busca pela cirurgia segura e, sobretudo, para introduzir a cultura de segurança do paciente no transoperatório, têm sido um grande desafio na atividade gerencial do enfermeiro. Com isso, a aplicação do checklist é a ferramenta de comunicação estruturada mais utilizada; ele é indispensável para mudar a cultura organizacional para que toda a equipe assistencial e de gestão compreenda a necessidade e os benefícios de sua realização (Fonseca et al., 2014; Jordão et al., 2019; Martins \& Dall'Agnol, 2016; Santos et al., 2018; Silva et al., 2017).

O tipo de anestesia escolhido pelo cirurgião está relacionado à técnica cirúrgica utilizada e ao emocional do paciente, pois pacientes ansiosos, que sofrem de crises de pânico, não suportam ficar despertos durante o ato cirúrgico e necessitam de sedação associada ao bloqueio ocular. Em muitos casos, as técnicas anestésicas mais utilizadas são: retrobulbar, peribulbar, subtenoniana e tópica. A anestesia tópica é utilizada como primeira opção por reduzir os riscos de possíveis complicações e propiciar alívio da dor (Moreira et al., 2019).

A sala de recuperação pós-anestésica é destinada a receber pacientes submetidos a procedimento anestésico-cirúrgico, tendo como finalidade proporcionar condições estruturais e funcionais durante o período de permanência até que recuperem seus reflexos, sua consciência e sinais vitais estabilizados dentro do padrão basal de cada paciente. No período em que o paciente permanece na sala de recuperação, o enfermeiro realiza uma avaliação criteriosa dos parâmetros basais e monitora as intervenções. O cuidado humanístico e a comunicação efetiva dos enfermeiros junto à implementação dos cuidados propiciam a satisfação dos pacientes e reduzem o risco de eventos adversos (Bonetti et al., 2017; Luo et al., 2015).

Para que o paciente possa realizar os cuidados no domicílio, são necessários a compreensão e o acompanhamento do enfermeiro. Com a alta hospitalar, os cuidados até então desempenhados pela equipe de Enfermagem passam a ser atribuídos e realizados pelo próprio paciente ou familiar. Para que o aprendizado e o entendimento sejam eficazes, a transição do cuidado deve ocorrer de forma clara e segura. Com isso, o papel de educador do enfermeiro torna-se fundamental para o sucesso desses cuidados.

Um plano de intervenções deve ser traçado em conjunto com a equipe multidisciplinar, composta por médico cirurgião, anestesista e enfermeiro, dando ênfase aos cuidados específicos do período de pós-operatório, como curativos, uso de medicações, retorno para acompanhamento e repouso domiciliar. O paciente deve saber onde procurar auxílio em caso de intercorrências e, principalmente, sentir-se seguro no momento da alta hospitalar para desempenhar esses cuidados (Araújo, \& Cassiolato, 2019; Pereira et al., 2018).

\section{Considerações Finais}

Este estudo tem o intuito de compartilhar e fortalecer ações voltadas à assistência e segurança ao paciente submetido a procedimentos oftalmológicos, trazendo o enfermeiro como facilitador do processo cirúrgico. Através das experiências 
vivenciadas pelas enfermeiras, em um centro cirúrgico ambulatorial na admissão, preparo, trans e pós-operatórios dos pacientes é possível aprimorando e contribuir com a aplicação dos conhecimentos de Enfermagem em Oftalmologia.

Desta forma, expondo seus desafios, preocupações e sentimentos, implementando uma assistência embasada em estratégias e associando o conhecimento técnico-científico, cumprindo os padrões de qualidade e segurança ao paciente, qualificando a assistência prestada aos cuidados de Enfermagem, sistematizando a assistência à saúde, estimulando o desenvolvimento do raciocínio crítico relacionado à temática.

\section{Referências}

Araújo, M. B., \& Cassiolato, H. M. P. (2019). Implementação da assistência de enfermagem ao indivíduo submetido a cirurgia oftalmológica de vitrectomia: relato de caso. Revista Científica Coorte, 9, 95-103.

Bonetti, A. E. B., Girardello, D. T. F., Coneglian, A. L. A., Egevardt, D., Batista, J., \& Cruz, E. D. A. (2017). Assistência da equipe de enfermagem ao paciente em sala de recuperação pós-anestésica. Revista de Enfermagem da UFSM, 7(2), 193-205.

Carvalho, A. F. A., Silva, R. B. V., \& Ferreira, E. B. (2016). Cirurgia de catarata pela técnica de facoemulsificação: um estudo de caso. Revista da Universidade Vale do Rio Verde, Minas Gerais, 14(1), 741-748.

Costa, D., Maltez, F., Mota, L., \& Príncipe, F. (2021). Relevance of information when elderly returning home after cataract surgery: nurses’ perspective. Millenium, 2(14), 21-28.

Daltro, M. R., \& Faria, A. A. (2019). Relato de experiência: uma narrativa científica na pós-modernidade. Estudos e Pesquisas em Psicologia, $19(1), 223-237$.

Fonseca, A. S., Peterlini, F. L., \& Costa, D. A. (2014). Segurança do Paciente. São Paulo: Martinari.

Jordão, K. M. D., Soares, R. A. Q., Fernandes, I. T. G. P., Nascimento, A. L., Ferreira, M. Z. J., \& Santos, S. M. (2019). Atuação do enfermeiro nos protocolos de cirurgia segura. Saúde Coletiva, Barueri, 9(49), 1538-1544.

Luo, S., Wang, Y., Li, Z., Ynag, C., \& Chen, A. (2015). Establishment and implementation of humanistic nursing care in ophthalmic ward. Eye Science, 30(3), $125-127$.

Martins, F. B., \& Dall'Agnol, C. M. M. (2016). Centro cirúrgico: desafios e estratégias do enfermeiro nas atividades gerenciais. Revista Gaúcha de Enfermagem, $37(4)$, e56945.

Meirelles, M. G. B., Santana, T. S., Vieira, L. T. Q., Costa, C. S. C., Celestino, K. A. A., Abud, M. B., \& Ávila, M. P. (2020). Prevalência das complicações da cirurgia de catarata em campanha assistencial. Brazilian Journal of Development, 6(7), 53783- 53790.

Moreira, F. G., Silva, L. R. F., Saraiva, F. P., \& Valbon, B. F. (2019). Study on immediate postoperative pain following cataract surgery: intraoperative endovenous administration of dipyrone. Revista Brasileira de Oftalmologia, 78(2), 98-102.

Muniz, R. V., \& Paczek, R. S. (2021). Melhores práticas de enfermagem/Enfermagem em cirurgias oftalmológicas: Moriá.

Oliveira, I. P. R. M., \& Meneguin, S. (2019). Resolubility of eye care in a secondary care center: a quantitative approach. Einstein, $17(3)$, eGS4385.

Pereira, J. S. P., Pinto, J. M. O., \& Castro, D. A. G. (2018). A influência da orientação da enfermagem antes da alta hospitalar no prognóstico do paciente. Revista Amazônia Science \& Health, 6(4), 8-12.

Ribeiro, I. C. A., \& Cunha, K. C. S. (2018). Evaluación del clima de seguridad del paciente en un hospital quirúrgico oftálmico. Enfermería Global, 17(52), $316-364$.

Ribeiro, I. C. A. (2017). Cuidado cirúrgico seguro em oftalmologia: cuidado cirúrgico seguro em oftalmologia: adaptação e operacionalização do checklist de segurança cirúrgica [Mestrado em Saúde e Tecnologia no Espaço Hospitalar, Universidade Federal do Estado do Rio de Janeiro]. Repositório da Biblioteca Virtual em Saúde.

Santana, T. S., Castro, V. A., Sousa, A. C., Siqueira, S. C., Vieira, L. T. Q., Faria, I. C. F., Vieira, J. G., \& Guimarães, J. G. (2020). Análise do atendimento e satisfação dos pacientes submetidos ao procedimento cirúrgico oftalmológico em um hospital público de Goiás. Brazilian Journal of Development, 6(7), 53746- 53754.

Santos, K. C. B., Feitosa, A. H. C., Ribeiro, G. S. C., \& Cavalcante, T. B. (2018). Metodologia da problematização com Arco de Maguerez no centro cirúrgico oftalmológico de um hospital universitário. Revista Ciência \& Saberes, 4(1), 884-888.

Silva, A. S., Silva, W. L. C., Gouvêa, M. A., \& Torres, E. L. (2017). Lista de verificação para cirurgia segura: barreiras para sua implementação em um serviço de oftalmologia. Revista de Gestão em Sistemas de Saúde, 6(3), 245-252.

Souza, I. T., Tenório, H. A. A., Gomes Júnior, E. L., Sarmento Neto, M. L., \& Almeida, B. R. M. (2019). Percepção do cliente no perioperatório sobre o cuidado de enfermagem no centro cirúrgico. REAS/EJCH, 26, e860. 